\title{
La survivance d'un système électoral sous le Consulat et l'Empire
}

Josiane Bourguet-Rouveyre

\section{Q OpenEdition \\ 1 Journals}

Édition électronique

URL : https://journals.openedition.org/ahrf/7473

DOI : 10.4000/ahrf.7473

ISSN : 1952-403X

Éditeur :

Armand Colin, Société des études robespierristes

Édition imprimée

Date de publication : 1 décembre 2006

Pagination : 17-29

ISSN : 0003-4436

Référence électronique

Josiane Bourguet-Rouveyre, "La survivance d'un système électoral sous le Consulat et l'Empire ",

Annales historiques de la Révolution française [En ligne], 346 | Octobre/Décembre 2006, mis en ligne le

01 décembre 2009, consulté le 22 avril 2022. URL : http://journals.openedition.org/ahrf/7473 ; DOI :

https://doi.org/10.4000/ahrf.7473

Ce document a été généré automatiquement le 22 avril 2022.

Tous droits réservés 


\title{
La survivance d'un système électoral sous le Consulat et l'Empire
}

\author{
Josiane Bourguet-Rouveyre
}

1 Au moment d'aborder la période du Consulat et de l'Empire, le général Mathieu Dumas - l'un des rapporteurs du décret créant la Légion d'honneur - écrit dans ses Souvenirs, à propos de la prise du pouvoir par Napoléon Bonaparte :

«Secondé par les chefs de l'armée, et sûr de l'obéissance des soldats, il osa frapper le coup d'État du 18 brumaire, et prit, avec le titre de premier Consul, les rênes du gouvernement de la République. Il maintint les formes apparentes de la représentation nationale dans le pouvoir législatif; mais, en interdisant aux deux chambres toute discussion publique, en imposant à la tribune et à la presse un silence absolu, il ne conserva que la dénomination chimérique et la vaine image d'un gouvernement républicain $»^{1}$.

2 Si l'on met de côté l'idée selon laquelle Bonaparte, dans cette affaire, aurait eu le soutien indéfectible de l'armée et se serait montré d'une résolution inébranlable, on ne peut que louer la lucidité de ce militaire libéral, ami de La Fayette et de la monarchie constitutionnelle, élu à l'Assemblée législative en 1791, puis aux Anciens, sous le Directoire, mais «fructidorisé » en l'an V pour n'avoir pas su se convaincre des bienfaits de la république, puis rallié à l'Empire sans obséquiosité mais avec une constance et une conviction qui le rendront sensible à l'appel des Cent-Jours. Ainsi la carrière et les convictions politiques de Mathieu Dumas me paraissent-elles résumer, presque à elles seules, les ambivalences d'un régime qui sut préserver les formes républicaines, tout en offrant à nombre de monarchistes constitutionnels un motif de ralliement. Vaine image d'un gouvernement républicain, selon notre auteur, le régime napoléonien fut ainsi parce qu'il ne sut pas conjuguer les formes de la représentation nationale, le système électoral, avec l'exercice des libertés politiques, dont Mathieu Dumas, qui écrit sous la Monarchie de Juillet, fait ici la pierre de touche de toute république. 
3 On pourrait assez facilement démontrer qu'en matière d'élections ou de plébiscites, le régime napoléonien n'invente rien. Depuis les élections aux États généraux, les Français ont été souvent appelés aux urnes, soit dans le cadre d'élections nationales, soit dans celui d'élections locales, le terme « aux urnes » étant ici pris dans un sens générique et masquant en réalité des pratiques très diverses, qui vont du vote recueilli oralement en assemblée, par assis-debout ou par acclamation, au vote par écrit, sur bulletin, qui ne constitue, pendant la décennie révolutionnaire, qu'une méthode parmi d'autres, comme l'ont montré les auteurs de l'ouvrage collectif Voter, élire pendant la Révolution française ${ }^{2}$.

4 Le corps électoral, pendant cette période a connu des variations en accordéon, depuis les démocratiques, mais aussi très inégales élections aux États généraux, régies par le code électoral de Necker. La règle a été ensuite, le plus souvent, le suffrage censitaire plus ou moins large, et même pour le temps de ce qu'il est convenu d'appeler la «République jacobine », Serge Aberdam a montré qu'il était illusoire de parler, dans la pratique, de suffrage universel masculin; le terme de "suffrage élargi » convenait mieux, en effet, aux pratiques électorales présidant aux élections locales et au plébiscite d'adhésion à la Constitution de $1793^{3}$. On sait que la Convention est élue par moins de 800000 citoyens et que le suffrage à deux degrés, maintenu pendant toute la période révolutionnaire, favorise la représentation des notables et permet d'amortir, sur le plan législatif, les aléas de l'expression populaire.

5 Le régime napoléonien n'a pas inventé le plébiscite. Ce mode de consultation populaire a été utilisé précédemment à deux reprises, une première fois, en 1793, pour l'adoption d'un texte constitutionnel qui ne sera jamais appliqué, la seconde fois, en 1795, pour ratifier la constitution du Directoire, texte accompagné du fameux décret dit des « deux tiers », par lequel on mettait d'emblée les premières élections législatives sous le signe de la candidature officielle, puisque les Conseils devaient se composer aux deux tiers d'anciens conventionnels ${ }^{4}$.

6 Il est d'usage de souligner le manque de fiabilité des résultats des plébiscites de l'époque consulaire, en dénonçant les façons peu délicates dont le Ministère de l'Intérieur avait usé, particulièrement sous Lucien Bonaparte, pour gonfler des chiffres jugés insuffisants pour asseoir la popularité du régime. Les travaux de Claude Langlois ont montré que le plébiscite de 1800 avait mobilisé moins de $25 \%$ du corps électoral, en supposant celui-ci fondé sur le suffrage universel masculin, preuve de l'attentisme d'une opinion échaudée par les expériences plébiscitaires antérieures ou les manœuvres électorales, et soucieuse de juger le régime à ses actes 5 . Ce pourcentage est inférieur à celui qu'on attribue généralement au plébiscite constitutionnel de 1793, crédité d'environ $30 \%$, ce qui est considéré comme un succès dans les circonstances du moment, mais pour lequel la comptabilité reste aléatoire, le mode d'expression des vœux n'étant pas uniforme, puisqu'on avait voté tantôt par écrit, tantôt oralement par acclamations, ou encore par assis-debout.

7 Et c'est peut-être ici que commencent les différences entre la période proprement révolutionnaire et celle du Consulat et de l'Empire. En effet, l'époque napoléonienne est marquée par une volonté nettement affichée d'uniformiser les modes d'expression électorale. En 1800, 1802 et 1804, les vœux des citoyens sont recueillis sur des registres. Certes, le système pêche encore par le fait que ces registres sont disposés dans des lieux très divers, et que les maires, les juges de paix, les hommes de loi et les divers fonctionnaires sollicités, s'acquittent de leur tâche avec un zèle variable et dans des 
formes également variables. Le recueil systématique des votes par écrit n'est pas sans danger pour le régime, car il ne peut plus être question, comme cela a pu se produire antérieurement, de faire un décompte avantageux et globalisant des votes favorables quand ceux-ci ont été recueillis en assemblée. Les registres sont là, qui font foi. Cela explique pourquoi on a pu considérer le plébiscite de l'an VIII comme un modèle du genre : la centralisation des résultats dans les bureaux du Ministère de l'Intérieur ayant montré une participation insuffisante, on sait comment Lucien Bonaparte arrondit les chiffres des départements et y ajouta les votes de l'armée censée avoir voté par acclamation. Ces manipulations seront renouvelées, mais dans une proportion nettement moindre, en 1802 et 1804 . Selon une estimation faite par Malcolm Crook, à partir des études les plus récentes consacrées à la fraude électorale, les plébiscites de 1800 et de 1815 peuvent être mis sur le même plan, avec une participation réelle qui n'aurait pas dépassé $22 \%$, alors que ceux de 1802 et 1804 sont un incontestable succès pour le régime, avec respectivement 47 et $42 \%$, scores nettement supérieurs à ceux enregistrés pendant la Révolution pour des consultations de même type ${ }^{6}$.

Il est donc assez manichéen d'opposer - comme on le fait trop souvent - le " plébiscite » napoléonien au « referendum » révolutionnaire et républicain, en laissant entendre que, dans le premier cas, il s'agirait d'une grossière manipulation destinée à légitimer un régime, et dans le second cas une authentique consultation populaire. Car en 1793 comme en 1795, il s'agit bien aussi, par le biais de la consultation, de légitimer un régime. En 1793, le projet montagnard de constitution a été élaboré hâtivement et s'inspire en partie, du projet dit " girondin » de Condorcet, lequel est dénoncé et jeté en prison, au moment même où commence la consultation. Celle-ci vise donc surtout à légitimer le coup de force du 2 juin 1793 et, alors que la révolte fédéraliste menace, à ressouder la nation autour de ce qu'il reste de la Convention ${ }^{7}$. Le texte constitutionnel, qui introduit un peu de démocratie directe dans l'adoption de la loi, en valorisant le rôle des assemblées primaires, peut rallier aussi bien les révolutionnaires prononcés de la sans-culotterie parisienne ou provinciale, que ceux qui ont dénoncé la dictature parisienne et réclamé plus d'autonomie locale. Quant aux députations envoyées à Paris pour le 10 août 1793, elles ont le double avantage de légitimer à la fois l'insurrection qui a inauguré la République et celle qui lui a permis de se débarrasser des impurs (les Girondins). Mais la question reste entière de savoir si les promoteurs de cette constitution avaient jamais eu l'idée de l'appliquer.

Les auteurs de la constitution de l'an III ne pouvaient faire moins que de soumettre leur texte, à leur tour, aux vœux de la nation. Thermidoriens, ils avaient, eux aussi, à justifier le coup de force par lequel ils avaient renversé Robespierre. Ils devaient également justifier le maintien provisoire d'une législation d'exception pour juger les "terroristes ", ainsi que l'enterrement définitif de la constitution de 1793, pourtant plébiscitée. Mais la situation de 1795 ressemble aussi, par bien des côtés à celle de l'an VIII. Dans les deux cas, le souci est celui de la stabilisation, en mettant un terme à une longue période de troubles civils. En 1795, c'est par une constitution supposée équilibrée et censitaire, de façon à se trouver aussi éloigné de l'aristocratie que de la démocratie; dans le cas du Consulat, il s'agit d'une sorte de dictature républicaine, où les formes sont conservées, mais les choix très contrôlés par l'intermédiaire du système des candidatures qui permet au pouvoir exécutif et au Sénat de composer à volonté les organes administratifs et législatifs. 
10 La constitution de l'an VIII a défini le corps électoral comme l'ensemble des citoyens, soit les hommes nés et résidant en France, âgés de 21 ans ou plus, et inscrits sur les registres civiques. Mais le système des listes de notabilité proposé par Sieyès, partiellement mis en œuvre en 1801, fonctionne mal. Rendant compte de la désignation des notables communaux de Valence, le préfet de la Drôme, Descorches, écrit ceci au ministre de l'Intérieur :

«On a peu voté; il n'a fallu que 9 suffrages pour devenir notable; la grande majorité des citoyens, dans les campagnes surtout, ne comprenait pas assez bien ce dont il s'agissait pour s'y livrer. L'expérience a rendu méfiants jusqu'à la défiance tous ces demi-aveugles. Ils veulent bien reconnaître à présent où ils poseront le pied, avant que d'aller en avant. [...] Il y a de nombreuses irrégularités dans les opérations de détail, mais la plus grande partie ne peut être justement attribuée qu'à la nature même de ces opérations compliquées, laborieuses "».

11 Ces difficultés qui soulignent le désintérêt pour la chose publique, particulièrement dans les campagnes, alertent le gouvernement. La création des collèges électoraux doit permettre, selon le Premier Consul, de donner plus de part au peuple dans les élections, en réduisant les degrés de sélection des notables ${ }^{9}$. Avec cette réforme, en effet, l'assemblée primaire, réunie au chef-lieu de canton, retrouve la place qu'elle avait sous la Révolution, comme base de la pyramide électorale, puisque, en son sein, sont choisis les candidats aux justices de paix et aux fonctions d'administration locale (il s'agit dans ce cas des conseillers municipaux) et qu'elle désigne les membres des collèges d'arrondissement et de département. Un cens n'est exigé que pour faire partie du collège de département, avec la mise en œuvre des listes des plus imposés.

Dans la pratique, les assemblées de canton sont loin de grouper l'ensemble des citoyens. En 1801, en l'absence de registres civiques, seuls ont été convoqués, pour la constitution des listes de notables communaux, ceux qui avaient le droit de vote sous le Directoire, mais, comme on l'a vu, la participation est faible et l'attentisme à l'égard du nouveau régime est la règle. La participation est meilleure à partir de 1803, sous le nouveau régime électoral, mais la mise à jour des registres civiques, commencée en 1791 et jamais achevée, exige des administrateurs locaux des efforts importants. Un long travail de recensement et de vérification est demandé aux maires qui n'ont pas toujours les compétences exigées ni le temps disponible. En 1806, ce travail est finalement confié aux sous-préfets et, désormais, l'inscription sur le registre est exigée de tout citoyen désirant participer à une assemblée électorale ${ }^{10}$.

13 Contrairement à ce qu'on écrit et dit encore parfois, le système électoral napoléonien a fonctionné. Les départements étant groupés en cinq séries, une série était convoquée chaque année ${ }^{11}$. Il s'ensuit que, sous le Consulat et l'Empire, assemblées cantonales et collèges électoraux se sont réunis au moins deux fois, et quelques-uns en 1813 pour une troisième fois. Toutes ces réunions ont fait l'objet de procès-verbaux réunis par les services préfectoraux, qui forment un corpus impressionnant de la sous-série F1cIII aux Archives nationales. Ces procès-verbaux témoignent de l'intérêt porté à ces procédures, pourtant formelles et démunies de tout ce qui pourrait constituer une campagne électorale. Comme sous la Révolution, le rôle des assemblées cantonales et des collèges électoraux se borne à l'élection, excluant toute délibération. Mais il faut y ajouter l'absence de débat dans la presse et l'inexistence de sociétés politiques susceptibles de peser sur les choix.

14 La procédure électorale est très exactement codifiée dans les instructions envoyées aux présidents des diverses assemblées au moment de leur convocation. Le président est 
tenu de prononcer un serment solennisant la session électorale, héritage révolutionnaire. Les réunions se tiennent nécessairement dans des lieux publics: préfecture, salle du conseil général ou d'arrondissement, hôtel de ville, mais parfois au théâtre, au musée ou à l'église, lorsque les édifices publics manquent. Au jour prévu, le bureau est formé : un secrétaire et des scrutateurs sont élus à la majorité des suffrages. Puis on procède aux opérations électorales proprement dites. Après le discours du président exhortant les participants à choisir des candidats bons pères de famille et dévoués à la fonction publique, sans mention de personnalités précises, il y a appel, puis rappel des participants. Le vote est toujours recueilli par écrit, sous forme de bulletins rédigés pendant l'assemblée et déposés dans autant d'urnes qu'il y a de catégories de candidats à désigner. L'usage strict des urnes est codifié depuis la loi du 19 fructidor an X (6 septembre 1802) : celles-ci doivent être de forme cubique, en bois et comporter une ou plusieurs serrures. Tous les bureaux de vote en seront dotés. Ces modalités sont rappelées à chaque convocation ${ }^{12}$.

15 À partir de 1806, les assemblées de canton sont divisées en sections afin de faciliter les opérations électorales, mais aussi de favoriser la participation, en évitant des réunions trop nombreuses et trop longues qui, auparavant, pouvaient se prolonger sur plusieurs jours, circonstance peu goûtée des ruraux, contraints de séjourner alors au chef-lieu de canton, avec tous les frais que cela pouvait entraîner. Le règlement électoral est alors rappelé dans une circulaire :

«Au jour fixé pour l'ouverture de la session, le président se rendra, au lever du soleil, dans l'édifice désigné par le préfet pour la réunion de la section. Sur le bureau, il y aura autant de boites que de fonctions diverses pour lesquelles l'assemblée sera chargée de faire des choix. Chacune de ces boites sera à double serrure; l'une des clés restera entre les mains du président; le plus imposé des scrutateurs aura l'autre. La salle devra être garnie d'un nombre de sièges et de tables suffisant pour que les votants puissent, sans confusion, former leurs bulletins. Du papier coupé de façon uniforme, de l'encre et des plumes seront déposés sur chaque table pour la formation des bulletins ${ }^{13}$.

16 Il est rappelé au président que «l'assemblée est votante et non délibérante». Une circulaire de 1810 rappelle encore que l'inscription sur le registre civique «ne se rattache au paiement d'aucune contribution ${ }^{14}$.

Sous l'Empire, le système électoral s'est donc installé dans des formes très codifiées permettant une appréciation statistique relativement fiable de la participation électorale. Le flou qui avait pu exister pendant la décennie révolutionnaire du fait que beaucoup de votes étaient exprimés oralement, disparaît. En témoignent les tableaux que les préfets sont chargés de dresser pour le Ministère de l'Intérieur, à partir de 1806, répertoriant par canton le nombre d'habitants et le nombre de votants, et mentionnant les opérations électorales à effectuer. Si les membres des collèges électoraux et les sénateurs sont nommés à vie, et les conseillers municipaux désignés pour 20 ans, les conseillers d'arrondissement et de département sont renouvelables, et pour cela, les collèges électoraux d'arrondissement et de département doivent désigner de nouveaux candidats ou reconduire les anciens.

18 À partir des procès-verbaux de réunion, il est donc possible d'apprécier, d'une façon assez juste, la participation électorale. Pour la Drôme, la session électorale s'ouvre à partir de septembre 1809 pour les cantons (il s'agit alors du renouvellement des justices de paix), et se prolonge jusqu'à la fin de décembre pour les collèges électoraux. Les résultats du canton de Châtillon sont les suivants: sur 1347 inscrits, il y a eu 384 
votants, soit une participation électorale de $28.5 \%$. Ces votants se sont répartis dans les diverses sections du canton. Pour la section de Châtillon, il y a eu 59 votants, parmi lesquels 25 illettrés ont dû faire appel aux services des scrutateurs pour écrire leur bulletin. Parmi ces illettrés, on trouve 23 propriétaires (on peut supposer qu'il s'agit majoritairement d'agriculteurs), un bottier et un tisseur de toile; parmi les autres votants se trouvent encore 18 propriétaires, un notaire, un receveur d'impôts, le juge de paix du canton, un percepteur, 2 ecclésiastiques et quelques artisans : 2 cordonniers, 2 maréchaux-ferrants, 1 menuisier et 1 tailleur. Tous ont inscrit ou fait inscrire leurs noms et qualités sur la feuille de présence ajoutée au procès-verbal ${ }^{15}$.

La participation électorale est plus élevée dans les collèges d'arrondissement et de département: elle oscille entre 60 et $75 \%$ des inscrits, pour l'ensemble des départements, selon J. Y. Coppolani ${ }^{16}$. Les enjeux électoraux ne sont certes pas considérables, puisque les sénateurs sont nommés à vie et que seul le renouvellement du Corps législatif et des conseils d'arrondissement et de département peut donner quelques espérances de représenter la nation ou de siéger dans des instances locales. Pourtant les notables continuent à porter un intérêt certain à ces opérations, ce qu'attestent non seulement le niveau élevé de participation, mais également l'existence d'une compétition entre candidats potentiels.

La création des collèges électoraux a modifié, plus qu'on ne le pense souvent, le système fondé sur les listes de notabilité, et cela sur deux points principalement: d'abord leur mise en œuvre à partir de véritables registres civiques régulièrement mis à jour, ensuite la désignation des candidats, non plus par un scrutin de liste à majorité relative, mais par un scrutin majoritaire à trois tours, aspect bien mis en lumière dans la thèse récemment publiée de Philippe Tanchoux ${ }^{17}$.

21 Voici l'exemple de l'assemblée électorale de l'arrondissement de Valence. L'élection de deux candidats au Corps législatif, commencée le 26 décembre 1809, ne se termine que le lendemain. Il y a 149 votants sur 198 inscrits, soit une participation de 75,25\%. Les deux premiers tours ne parviennent pas à départager les quatre notables désignés par les votes des électeurs, car la majorité absolue est exigée pour être élu (les bulletins doivent, à chaque tour, comporter deux noms); il faut donc un troisième tour pour désigner les candidats à la majorité relative : les deux élus ont 79 et 76 voix, les deux perdants 72 et 66 voix. Il y a donc bien eu compétition, dans un scrutin serré18.

La session du collège de département qui se tient à Valence est moins palpitante, l'élection des candidats au Sénat ayant désigné sans surprise le comte de Montalivet, à l'unanimité, et un conseiller d'État, à la majorité absolue, dès le premier tour (Montalivet est ministre de l'Intérieur depuis le 1er octobre). Par contre, il faut trois tours pour désigner les candidats au Corps législatif. Il y a eu 152 présents sur 209 inscrits, soit une participation de $72.7 \%^{19}$.

23 Fait notable, le collège de département a tenu à envoyer une adresse et une députation à l'empereur. Ces pratiques, qui ont accompagné l'époque révolutionnaire, n'ont pas cessé sous le régime napoléonien. Sous le Consulat, elles revêtent même un caractère spontané, non codifié par le pouvoir; des initiatives locales permettent de clore les assemblées électorales sur une note consensuelle. Adresses et députations deviennent systématiques à partir du sacre, au point que le gouvernement finit par en fixer les formes par le décret du 26 novembre 1808. Or, la rédaction de l'adresse et l'élection de la députation font l'objet d'un court débat à l'intérieur de l'assemblée électorale. C'est ce que montre le procès-verbal de l'assemblée de Valence : 


\begin{abstract}
«Monsieur le président a soumis à la décision de l'assemblée la proposition suivante : la rédaction du projet d'adresse sera-t-elle confiée à une commission de six membres, choisis dans le sein du collège à la pluralité relative des suffrages? Il a prévenu l'assemblée que si cette proposition n'était pas adoptée, il resterait chargé de plein droit de la rédaction de l'adresse.

L'assemblée ayant décidé, par assis et levé, qu'elle n'adoptait pas la proposition, il a été procédé de suite, dans la forme prescrite, à la nomination d'une commission de cinq membres pour coopérer, avec Monsieur le président, à la rédaction du projet d'adresse [...] L'assemblée a également arrêté, sur la proposition de Monsieur le président, que la députation serait composée de cinq membres, et que le scrutin pour la nomination des députés commencerait de suite et resterait ouvert pendant tout le temps que dureraient ces opérations $»^{20}$.
\end{abstract}

Le scrutin a lieu par écrit. Le dépouillement désigne le président comme chef de la délégation, et avec lui, un magistrat, deux propriétaires et le maire d'une commune du département. Ensuite l'adresse est lue et adoptée par l'assemblée. Cette adresse très respectueuse insiste sur l'exactitude avec laquelle les notables départementaux s'acquittent de tous leurs devoirs: "Notre jeunesse se range avec zèle sous vos drapeaux, nos charges publiques s'acquittent avec exactitude », mais elle loue aussi l'empereur de ce qu'il a su, par sagesse, ne pas imposer aux habitants de nouvelles charges, ce qui est peut-être une façon détournée de souligner la lourdeur de celles qui pèsent déjà sur eux. Tout au long du procédé par lequel l'adresse et la députation ont été mises en œuvre, le rôle du président, nommé par le gouvernement, a été déterminant, mais on constate qu'il a cherché à associer à son initiative les membres du collège, par trois votes successifs.

Il y a parfois des accrocs à la bonne ordonnance des assemblées électorales. Lorsque la session s'ouvre pour l'arrondissement de Nyons, le 19 décembre 1809, le quorum est tout juste atteint, avec 58 participants sur 113 inscrits (51.3\%). À l'issue de la première journée, les candidats au Corps législatif ont pu être désignés au bout de trois tours, mais, le lendemain, les électeurs que " leurs affaires et l'inconstance du temps ", dit le procès-verbal, forcent à se retirer, sont en nombre insuffisant pour poursuivre les opérations qui doivent désigner les suppléants. Il faut attendre six jours avant que le quorum soit à nouveau atteint et, pendant ce temps, le bureau siège en permanence. Il faudra à nouveau trois tours pour départager les candidats à la suppléance, puis on procèdera à l'élection des candidats au conseil d'arrondissement. Ici, l'enthousiasme a manifestement manquée ${ }^{1}$.

En comparant le système électoral napoléonien avec les pratiques de la première République, on aboutira donc à un bilan contrasté. La participation électorale, sous l'Empire, est plutôt meilleure que sous la République, sans doute parce qu'elle est mieux codifiée et encadrée. De surcroît, l'abondance des archives, sur ce sujet, la rigueur avec laquelle les services préfectoraux centralisent les procès-verbaux et totalisent les informations qui s'y trouvent, laissent peu de place à l'imagination du chercheur. Et ce n'est pas le moindre des paradoxes que de découvrir ces assemblées électorales de l'Empire, se réunissant avec une régularité remarquable, et procédant méticuleusement à de longues opérations qui ne mettent en compétition que des candidats à la candidature. Certes, la vie politique se concentre désormais à l'intérieur d'un groupe de notables satisfaits du régime tant que celui-ci leur assure la paix civile indispensable à la prospérité des affaires, sans accroître trop lourdement les charges collectives. De ce point de vue, la très modeste participation aux assemblées cantonales contraste avec l'assiduité dans les collèges électoraux ; mais on remarquera que ce n'est 
pas une tendance que le régime a voulu encourager, puisque la création des sections cantonales vise précisément à supprimer les contraintes qui pouvaient empêcher les ruraux, particulièrement, d'émettre des vœux. La popularité du régime doit se mesurer, non seulement au travers des plébiscites, mais également par la participation aux assemblées primaires. Il serait faux d'affirmer, par exemple, qu'après le sacre, Napoléon n'a plus besoin de preuves d'adhésion populaire. Tout au long de l'Empire, par conséquent, le régime a été conscient de la nécessité pour lui de conserver des formes de représentation le liant étroitement à la nation, formes héritées non seulement de la République, mais également de la monarchie constitutionnelle.

\section{ANNEXES}

Division des assemblées de cantons en sections et indication de l'espèce et du nombre de nominations qu'elles auront à faire à leur prochaine session

\begin{tabular}{|c|c|c|c|c|c|c|}
\hline Arrondissemerts & Cantons & Habitarts & Votants & \begin{tabular}{|c|} 
Candidats \\
arrondissement
\end{tabular} & \begin{tabular}{|c|} 
Candidats \\
département
\end{tabular} & Communes \\
\hline \multirow{9}{*}{ Die } & Bourdeaux & 4138 & 1066 & 8 & 4 & - \\
\hline & Châtillon & 6128 & 1347 & 14 & 7 & - \\
\hline & Crest-nord & 12819 & 2763 & 23 & 11 & - \\
\hline & Crest-sud & 7396 & 1665 & 15 & 7 & - \\
\hline & Die & 6968 & 1623 & 16 & 8 & - \\
\hline & $\begin{array}{l}\text { La Chapelle } \\
\text { en Vercors }\end{array}$ & 5043 & 872 & 10 & 5 & - \\
\hline & $\begin{array}{l}\text { LaMotte } \\
\text { Chalençon }\end{array}$ & 7747 & 1722 & 16 & 8 & - \\
\hline & \begin{tabular}{|l} 
Luc en Diois \\
\end{tabular} & 4545 & 1018 & 9 & 4 & - \\
\hline & Saillans & 4692 & 1116 & 9 & 4 & - \\
\hline Total & 48 sections & 59416 & \begin{tabular}{|l|}
13192 \\
\end{tabular} & 120 & 58 & - \\
\hline \multirow[t]{5}{*}{ Montélimar } & Dieulefit & 9498 & 2184 & 23 & 9 & - \\
\hline & Grignan & 8594 & 2089 & 20 & 8 & - \\
\hline & Marsane & 7141 & 1503 & 16 & 6 & - \\
\hline & Mortélimar & 10550 & 2813 & 28 & 10 & - \\
\hline & Pierrelatte & 13798 & 3311 & 33 & 12 & - \\
\hline Total & 39 sections & 49581 & 11900 & 120 & 45 & - \\
\hline \multirow[t]{4}{*}{ Nyons } & Le Buis & 9183 & 2900 & 36 & 9 & - \\
\hline & Nyons & 10631 & 2697 & 40 & 10 & - \\
\hline & Rémusat & 4331 & 1036 & 14 & 4 & - \\
\hline & Cédéron & 8058 & 1945 & 30 & 7 & - \\
\hline Total & 29 sections & 32204 & 7978 & 120 & 30 & - \\
\hline \multirow[t]{10}{*}{ Valence } & Bourg de Péage & 16585 & 3393 & 30 & 15 & - \\
\hline & Chabeuil & 11825 & 2566 & 22 & 11 & - \\
\hline & Le Grand Serre & 9609 & 1912 & 13 & 7 & $\cdot$ \\
\hline & Loriol & 7068 & 1608 & 22 & 6 & - \\
\hline & Romans & 17459 & 3373 & 30 & 15 & - \\
\hline & Saint-Donat & 5213 & 1043 & 10 & 5 & - \\
\hline & $\begin{array}{l}\text { Saint Jean } \\
\text { en Royans }\end{array}$ & 7135 & 1396 & 14 & 7 & - \\
\hline & Saint-Vallier & 10742 & 2029 & 20 & 10 & $\cdot$ \\
\hline & Tain & 7736 & 1722 & 15 & 8 & - \\
\hline & Valence & 14420 & 3275 & 18 & 14 & - \\
\hline Total & 72 sections & 107792 & 22317 & 194 & 98 & - \\
\hline Total département & 188 sections & 248993 & 54787 & 554 & 231 & - \\
\hline
\end{tabular}

Fait et arrêté par le préfet, à Valence, le 20 octobre 1806, en exécution du décret impérial du 17 janvier 1806. La colonne des communes est restée vide car le préfet n'a pas eu connaissance de vacances.

AN, F1cIII Drôme 3 


\section{NOTES}

1.Souvenirs du lieutenant général comte Mathieu Dumas, 1770-1836, publiés par son fils, Paris, 1839, tome III, p. 168.

2.Serge ABERDAM, Serge BIANCHI et alii, Voter, élire pendant la Révolution française, 1789-1799, Paris, CTHS, 1999. On peut lire, page 40 : «Le scrutin individualisé se pratique depuis les premières assemblées élisantes de la période révolutionnaire ; mais le vote secret par bulletins ne devient obligatoire qu'en 1795. Auparavant, des alternatives au vote secret étaient tolérées, notamment le vote à haute voix ». Les auteurs ajoutent que des pratiques « archaïques » ont perduré, notamment à l'occasion de la consultation sur le texte constitutionnel de 1793, comme le vote par acclamation.

3.Serge ABERDAM, Démographes et démocrates. L'œuvre du Comité de division de la Convention nationale, Paris, Société des études robespierristes, 2004.

4.Malcom Crook utilise le terme de " plébiscite " pour désigner les consultations populaires qui s'échelonnent entre 1793 et 1815, sans distinction; voir « The uses of Democracy. Elections and Plebiscites in Napoleonic France ", dans M. F. CRoss and D. WiLLiams (dir.), The French Experience from Republic to Monarchy, 1792-1824, Houndmills, Pallgrave, 2000, p. 58-71.

5.Claude LANGLOIS, « Le plébiscite de l'an VIII et le coup d'État du 18 pluviose an VIII », AHRF, 1972, p. 43-65, 231-246, 390-415.

6.Malcolm СRоOK, «Confiance d'en bas, manipulation d'en haut : la pratique plébiscitaire sous Napoléon ", dans Philippe BouRDIN et alii (dir.), L'incident électoral de la Révolution à la Ve république, Clermont-Ferrand, Presses universitaires Blaise Pascal, 2002, p. 77-87.

7.Voir l'analyse présentée par Ettore Rotelli (Faculté de Sciences politiques de Bologne) dans un ouvrage récemment paru : Forme di governo delle democrazie nascenti, 1689-1799, Bologne, Il Mulino, 2005. Il écrit, page 412 : « Consapevole del vizio d'origine, costituito della previa eliminazione dei convenzionali girondini, la nuova leadership montagnarda e giacobina, dal 2 giugno al potere ormai incontrastata, intende utilizzare il referendum per la propria legittimazione ».

8.AN, F1CIII Drôme 2, Descorches au ministre de l'Intérieur, 21 thermidor an IX.

9.On sait que le Premier Consul s'était montré dès le début méfiant à l'égard du système préconisé par Sieyès : «Dans le système des listes de notabilité, le peuple qui présente en définitive cinq mille candidats ne peut se flatter de concourir assez aux élections, pour voir nommés ceux qui ont le plus sa confiance. Pour la stabilité du gouvernement, il faut donc que le peuple ait plus de part aux élections et qu'il soit réellement représenté. Alors il se ralliera aux institutions ; sans cela, il y restera toujours étranger ou indifférent ", cité par A. C. THIBAUDEAU, Mémoires sur le Consulat, Paris, 1827, tome II, p. 289.

10.Josiane BOURGUET-ROUVEYRE, « La citoyenneté à l'épreuve du conformisme et de l'uniformité sous le Consulat et l'Empire ", dans Terminée la Révolution, IVe colloque européen de Calais, textes réunis par Michel BIARD, $\mathrm{n}^{\circ}$ hors série du Bulletin des Amis du Vieux Calais, 2002, p. 89-99.

11.Jean Yves CoppolanI, Les élections en France à l'époque napoléonienne, Paris, Éditions Albatros, 1980, p. 3-6; Philippe TANCHOUX, « Les procédures électorales sous le Consulat et l'Empire » dans Michel PERTUÉ et alii (dir.), Suffrage, citoyenneté et révolutions, 1789-1848, Paris, Société des études robespierristes, 2002, p. 43-73, et Les procédures électorales en France de la fin de l'Ancien Régime à la première guerre mondiale, Paris, CTHS, 2004 ; Annie 
BLÉTON-RUGET, « Ordre social, ordre politique et ordre territorial : les collèges électoraux de l'an X dans le système politico-administratif napoléonien ", dans Jean-Jacques CLèrEE et Jean-Louis HALPERIN (dir.), Ordre et désordre dans le système napoléonien, Paris, Éditions La Mémoire du droit, 2003, p. 119-134.

12.La loi du 13 ventôse an IX prescrit d'utiliser des boites fermant à clé, trois serrures sont même exigées pour les élections départementales et nationales. Le règlement du 18 fructidor an $\mathrm{X}$ en ordonne l'utilisation, de sorte que leur usage va se généraliser, malgré le coût élevé pour les communes, car il en faut plusieurs par bureau de vote. Pendant la Révolution, divers récipients avaient été utilisés, parmi lesquels des vases en métal ou en faïence, des paniers dont l'inviolabilité n'était garantie que par des cartons maintenus à l'aide de ficelles dont les nœuds étaient cachetés (Jean Yves Coppolani, op. cit., p. 201-203).

13.AN, F1CIII Drôme 3, Instructions pour Messieurs les présidents d'assemblées de canton.

14.AN, F1CIII Drôme 3, Décisions données par le ministre sur diverses questions qui lui ont été présentées. L'une de ces questions concernait les jeunes gens âgés de 21 ans accomplis et habitant chez leur parents : pouvaient-ils être inscrits sur les registres civiques? Montalivet répond : «Oui, d'après les dispositions des articles 2 et 6 de l'acte des constitutions de l'Empire, en date du 22 frimaire an VIII, d'après celle de l'article $2 \mathrm{du}$ règlement du 17 janvier 1806, l'inscription civique ne se rattache au paiement d'aucune contribution ».

15.AN, F1CIII Drôme 3, procès-verbal de Châtillon.

16.Jean Yves Coppolani, op. cit., pages 234 et suivantes.

17.Philippe TANCHOUX, op. cit.

18.AN, F1CIII Drôme 3, procès-verbal de l'assemblée électorale de l'arrondissement de Valence.

19.AN, F1CIII Drôme 3, procès-verbal de l'assemblée électorale du département à Valence.

20.Ibid.

21.AN, F1CIII Drôme 3, procès-verbal de l'assemblée électorale de l'arrondissement de Nyons. Le scrutin se déroule du 20 au 26 décembre 1809.

\section{RÉSUMÉS}

Il peut paraître paradoxal d'affirmer qu'en matière d'élections et de plébiscites, la période napoléonienne est l'héritière de la Révolution, tant on a souvent opposé le referendum révolutionnaire au plébiscite bonapartien, ou les formes démocratiques de participation à la vie politique sous la première République à la rigidité du système impérial. Pourtant l'étude du système électoral et des consultations populaires, sous le Consulat et l'Empire, montre bien une continuité avec la décennie révolutionnaire : le régime ne fait que codifier et uniformiser des pratiques apparues antérieurement et, comme sous la Révolution, l'assemblée de canton reste la base du système. L'absence de débat et de libertés publiques n'empêche pas la participation électorale ; celle-ci est même élevée dans les assemblées d'arrondissement et de département où 
se réunissent des notables. Même sous l'Empire, Napoléon a donc eu besoin de conserver des formes de représentation le liant étroitement à la nation, modèles empruntés aussi bien à la monarchie constitutionnelle qu'à la république.

The Survival of the Electoral System under the Consulate and Empire. It might seem paradoxical to suggest that on the issue of elections and plebiscites, the Napoleonic period is the heir of the Revolution, for the revolutionary referendum has often been contrasted with the Bonapartist plebiscite, and the democratic forms of political participation under the First Republic with rigidity of the Imperial system. But a study of the electoral system and popular consultations under the Consulate and Empire clearly shows a continuity with the revolutionary decade: the regime only codified and standardized earlier practices, and, as under the Revolution, the cantonal assembly remained the foundation of the system. The absence of debate and public liberties did not prevent electoral participation; rather, this is even higher in the assemblies of the arrondissement and the department where the notables met. Even under the Empire, Napoleon had to conserve forms of representation, tying them closely to the Nation, models borrowed as much from the Constitutional Monarchy as from the Republic.

INDEX

Mots-clés : nation, referendum, plébiscite, assemblées primaires, participation électorale, légitimation

\section{AUTEUR}

\section{JOSIANE BOURGUET-ROUVEYRE}

Université de Paris I, 17 rue de la Sorbonne

75231 Paris cedex 05, bourguet josiane@yahoo.fr 